\title{
Chapitre 4
}

\section{Transition 1 : Modéliser les migrations et la colonisation de nouveaux territoires par les Homo Sapiens}

Christophe Coupé, Dynamique Du Langage,UMR 5596 CNRS et Université Lyon2, 14, Avenue Berthelot 69363 Lyon Cedex 07.

Jean-Marie Hombert, Dynamique Du Langage, UMR 5596 CNRS et Université Lyon2, 14, Avenue Berthelot 69363 Lyon Cedex 07.

Florent Le Néchet, Université Paris-Est, Laboratoire Ville Mobilité Transport, UPEMLV : 5

boulevard Copernic, Cité Descartes F 77454 Marne-la-Vallée cedex 2 France

Hélène Mathian, univ. Lyon, CNRS, UMR 5600 EVS, ENS- 15 parvis René Descartes, F69342 Lyon cedex 7.

Lena Sanders, Géographie-cités, UMR 8504 CNRS

La première transition étudiée dans le cadre du programme TransMonDyn est la plus ancienne : il s'agit de la dispersion de notre espèce, Homo sapiens, à la surface de la planète. Cette expansion est marquée en particulier par la sortie hors d'Afrique de nos ancêtres : une première fois mais de façon circonscrite au Proche-Orient il y a environ 100000 ans, puis à nouveau quelques 30000 ans plus tard. Ces migrations conduisent notre espèce en Asie et en Europe, mais également à la conquête de terres auparavant inoccupées par les êtres humains : l'Australie et les Amériques.

Nous présentons ici cette transition, ainsi qu'une tentative de modélisation afin de mieux cerner les différents facteurs qui ont conditionné ses caractéristiques spatiales et temporelles.

Nous introduisons d'abord le contexte de la transition, ainsi que ses différentes dimensions et ses possibles causes. Ces éléments permettent d'éclairer le modèle que nous présentons ensuite. Il s'agit d'un modèle de simulation qui permet de tester des hypothèses sur la manière dont nos ancêtres ont peuplé, par migration, de nouveaux territoires. Nous spécifions les différents composants du modèle, et donnons enfin les résultats des simulations et quelques interprétations.

\section{Homo sapiens à la conquête de la Terre}

\section{L'émergence de l'Homme moderne dans le cadre de l'évolution humaine}

Il y a environ 150000 ans, l'apparition de l'Homme anatomiquement moderne, Homo sapiens, est la dernière étape d'un long processus évolutif, celui qui prend place au sein de la sous-tribu ${ }^{1}$ des Hominines suite à sa divergence avec la sous-tribu des Panines (à laquelle appartiennent les chimpanzés), il y a 7 à 8 millions d'années. Au cours de cette longue période, de nombreuses espèces se succèdent et parfois coexistent avant la naissance d'Homo habilis, il y a environ 2,6 millions d'années. Son nom et son statut de premier représentant du genre humain lui viennent des premiers outils de pierre auxquels il est classiquement associé, même si cette relation est aujourd'hui remise en question ${ }^{2}$. Suite à cette innovation culturelle

\footnotetext{
${ }^{1}$ En classification biologique, une sous-tribu est un taxon situé immédiatement au-dessus du genre.

${ }^{2}$ Harmand Sonia, Lewis Jason E., Feibel Craig S., Lepre Christopher J., Prat Sandrine, Lenoble Arnaud, Boës Xavier, et al., « 3.3-million-year-old stone tools from Lomekwi 3, West Turkana, Kenya », Nature, 521(7552), 2015, p. 310-315.
} 
majeure, près de 800000 ans s'écoulent avant qu'un successeur, Homo ergaster, ne quitte l'Afrique pour se répandre à travers l'Eurasie.

L'apparition de notre espèce est ainsi à replacer dans une évolution «buissonnante » dans l'espace et le temps. Les premiers Homo sapiens n'étaient en particulier pas les seuls êtres humains sur la planète, et ils ont côtoyé leurs « cousins » autant en Afrique que plus tard en Asie et en Europe, avant que ceux-ci ne disparaissent. La nature de leurs relations est encore mal comprise, mais depuis quelques années, l'analyse de patrimoines génétiques fossiles suggère que des métissages ont eu lieu entre Homo sapiens et deux autres espèces : les Neandertals et les Denisoviens ${ }^{3}{ }^{4}$. Les gènes ne nous renseignent toutefois pas sur la nature et la fréquence des contacts entre groupes d'espèces différentes. Si l'on commence à comprendre le rôle qu'ont pu jouer certains gènes en termes d'adaptation à l'environnement, l'existence de transmissions culturelles est loin d'être prouvée.

Les échelles de temps sont parfois trompeuses, et il importe de rappeler que l'apparition d'Homo sapiens est bien antérieure aux grandes innovations culturelles qui sont la marque de nombre de sociétés modernes. L'agriculture et la sédentarisation sont en effet apparues il y a environ 10000 ans, et l'écriture il y a « seulement » 6000 ans. Les premières peintures sont plus anciennes, mais même datées de près de 40000 ans, plus de 100000 ans les séparent des premiers de nos ancêtres directs. Pendant la plus grande partie de son existence, notre espèce a ainsi été caractérisée par des structures culturelles et démographiques bien différentes de celles que nous observons communément aujourd’hui.

\section{La théorie 'Out of Africa'}

La présence d’Homo sapiens sur la quasi-totalité des terres émergées a engendré de nombreuses théories sur les processus de peuplement liés à notre espèce. En ce qui concerne en particulier les épisodes les plus anciens de peuplement, la théorie multirégionale et la théorie Out of Africa se sont affrontées dans les années 1980, avant que la seconde ne prenne l'avantage.

La théorie multirégionale, initialement formulée en $1984^{5}$, s'oppose à l’idée que différentes espèces se distinguent au sein du genre humain. Au contraire, elle défend l'existence d'une unique espèce, apparue avant la première sortie hors d'Afrique, et qui regroupe les populations plus anciennes d'Homo erectus, d'Homo neandertalensis etc., et les populations plus récentes d'Homo sapiens. Le passage des populations archaïques à celles plus modernes se serait produit de façon continue au cours du temps, ce qui distingue cette théorie d'une conception polygénétique de l'origine des êtres humains modernes, c'est-à-dire d'une émergence en plusieurs lieux distincts ${ }^{6}$. La préservation d'une seule espèce au niveau global et l'existence de différences régionales entre ses représentants nécessite un équilibre entre des flux de gènes homogénéisateurs et des phénomènes locaux de dérive génétique et de sélection.

\footnotetext{
${ }^{3}$ Prüfer Kay, Racimo Fernando, Patterson Nick, Jay Flora, Sankararaman Sriram, Sawyer Susanna, Heinze Anya, et al., " The complete genome sequence of a Neanderthal from the Altai Mountains ", Nature, 505(7481), 2014, p. 43-9.

${ }^{4}$ Reich David, Green Richard E., Kircher Martin, Krause Johannes, Patterson Nick, Durand Eric Y., Viola Bence, et al., " Genetic history of an archaic hominin group from Denisova Cave in Siberia ", Nature, 468(7327), 2010, p. 1053-1060.

${ }^{5}$ Wolpoff Milford H., Wu Xin Zhi, Thorne Alan G., « Modern Homo Sapiens Origins: A General Theory of Hominid Evolution Involving the Fossil Evidence from east Asia ", The Origins of Modern Humans: A World Survey of the Fossil Evidence, New York, Liss, 1984, p. 411-483.

${ }^{6}$ Wolpoff Milford H., Hawks John, Caspari Rachel, "Multiregional, not multiple origins », American Journal of Physical Anthropology, 112(1), 2000, p. 129-136.
} 
A l'opposé d'une lecture " en continuité » de l'évolution humaine, la théorie de remplacement couramment appelée Out of Africa considère que les populations humaines en Eurasie ont été remplacées par une population plus moderne venue d'Afrique ${ }^{7}$. Notre espèce serait apparue en Afrique de l'est il y a entre 150000 et 200000 ans, avant de se répandre sur ce continent puis sur les autres le long de différentes voies migratoires ${ }^{8}$. Cette théorie a aujourd'hui l'aval de la majeure partie des spécialistes, et s'appuie à la fois sur des données paléoanthropologiques $^{9}{ }^{10}$ et des données génétiques ${ }^{11}{ }^{12}$. Les chercheurs de ces différentes disciplines cherchent aujourd'hui à préciser les routes de migrations empruntées, et les dates qui peuvent leur être associées. L'étude des distributions géographiques de différents haplogroupes ${ }^{13}$, en particulier portés par l'ADN mitochondrial et l'ADN du chromosome Y, pointent en particulier vers les mouvements migratoires de nos lointains ancêtres.

L'histoire qui se dessine aujourd'hui pour notre espèce est ainsi constituée d'anciennes migrations en Afrique et d'une possible première sortie précoce de l'Afrique en direction du Proche-Orient, sans toutefois dépasser cette région ${ }^{14}$. Ainsi, c'est une seconde sortie plus tardive, il y a environ 60000 à 70000 ans, qui marque le véritable début de l'expansion de notre espèce à travers l'Asie puis le reste du monde. L'observation d'un "goulet d'étranglement » génétique à cette période suggère une petite taille de population fondatrice ${ }^{15}$.

Suite à cette vague migratoire qui a conduit Homo sapiens en Asie du sud, en Asie du sud-est, en Australie et en Nouvelle-Guinée, la dispersion de notre espèce en Europe s’est faite quant à elle plus tardivement, il y a environ 40000 ans $^{16}$. La partie la plus orientale de l'Asie a été colonisée également à partir de cette période. L'est de la Béringie ${ }^{17}$ a en particulier été atteint

\footnotetext{
${ }^{7}$ Stringer Christopher B., "Modern human origins: progress and prospects ", Philosophical transactions of the Royal Society of London: Series B, Biological sciences, 357, 2002, p. 563-579.

8 Lahr Marta Mirazon, Foley Robert, "Multiple Dispersals and Modern Human Origins ", Evolutionary Anthropology, 3(2), 1994, p. 48-60.

9 Lahr Marta Mirazon, "The Multiregional Model of modern human origins: a reassessment of its morphological basis ", Journal of Human Evolution, 1994.

${ }^{10}$ White Tim D., AsfawBerhane, DeGusta David, Gilbert Henry, Richards Gary D., Suwa Gen, Howell, F. Clark, « Pleistocene Homo sapiens from Middle Awash, Ethiopia », Nature, 423(6941), 2003, p. 742-747.

${ }^{11}$ Cann Rebecca L., Stoneking Mark, Wilson Allan C., "Mitochondrial DNA and human evolution ", Nature, 325(6099), 1987, p. 31-36.

12 Underhill Peter A., Passarino Giuseppe, Lin Alice A., Shen P., Lahr Marta Mirazon, Foley Robert A., Oefner Peter J., Cavalli-Sforza Luigi Luca, « The phylogeography of $Y$ chromosome binary haplotypes and the origins of modern human populations ", Annals of human genetics, 65, 2001, p. 43-62.

${ }^{13}$ En anthropologie moléculaire, des gènes fortement liés les uns aux autres ont une forte probabilité d'être transmis ensemble au cours des générations. Cette héritabilité partagée et de rares mutations permettent de distinguer différentes populations sur la base de leurs gènes et d'étudier leur histoire. Dans ce cadre, un haplotype désigne un ensemble d'allèles (c'est-à-dire de variantes) de gènes transmis ensemble, et un haplogroupe un ensemble d'haplotypes qui permet de définir une population et un ancêtre commun à ses membres.

${ }^{14}$ Liu Hua, Prugnolle Franck, Manica Andrea, Balloux François, « A geographically explicit genetic model of worldwide human-settlement history ", American journal of human genetics, 79(2), 2006, p. 230-237.

${ }^{15}$ Liu H. et al., " A geographically explicit... », op.cit., p. 230-237.

${ }^{16}$ Mellars Paul, « Going East: New Genetic and Archaeological Perspectives on the Modern Human Colonization of Eurasia ", Science, 313(5788), 2006, p. 796-800.

${ }^{17}$ La Béringie est le passage terrestre qui reliait la Sibérie et l'Alaska lors de fortes baisses du niveau des mers.
} 
il y a environ 30000 ans $^{18}$ par des populations qui ont plus tard rapidement colonisé les Amériques, il y a un peu plus de 15000 ans $^{19}$.

Concernant l'expansion d'il y a environ 70000 ans, un débat existe quant à un passage par la corne de l'Afrique et la Mer Rouge et/ou une migration le long du Nil puis à travers le Levant, mais différentes recherches pointent plutôt en direction de la première voie : des données génétiques $^{20}$, mais aussi des découvertes archéologiques dans la péninsule arabique qui suggèrent une occupation il y a plus de $120000 \mathrm{ans}^{21}$. Suite à ce premier franchissement, les petits groupes de population auraient longé les côtes du sud de l'Asie pour atteindre l'Indonésie puis l'Australie il y a environ 50000 ans. Etablir avec confiance les anciennes routes de migration est difficile en particulier à cause des évolutions du climat et du niveau des mers au cours du dernier épisode glaciaire, qui a débuté il y a environ 120000 ans et a culminé il y a 21000 ans. L'expansion des glaces s'est traduite par une baisse importante du niveau des eaux : de $50 \mathrm{~m}$ ou $70 \mathrm{~m}$ il y a 70000 ans à $120 \mathrm{~m}$ lors du maximum glaciaire. Ceci a très probablement facilité le passage du détroit de Bab-el-Mandeb dans la Mer Rouge (voir figure 1), avec la présence alors d'îles aujourd'hui sous la surface. La colonisation du Sahul, le bloc terrestre qui reliait l'Australie et la Nouvelle-Guinée durant la majeure partie de la dernière ère glaciaire, est particulièrement significative. Plusieurs sites archéologiques en Australie permettent d'être certain de la présence d'Homo sapiens sur l'île il y a environ 50000 ans $^{22}$. L'étude de la paléographie de la région de Wallacea, entre le Sahul et le Sundanom donné aux terres émergées alors à l'extrémité sud-est de l'Asie - permet de conclure que plusieurs traversées maritimes ont été nécessaires, l'une d'elles atteignant au moins $90 \mathrm{~km}^{2324}$. La maîtrise de la navigation sous-tendue par ces longues et difficiles traversées a pu se développer progressivement le long de la route côtière menant de l'Afrique au Sahul, comme le suggère l'occupation très ancienne des îles Andaman dans l'Océan Indien, elle aussi nécessairement par voie maritime ${ }^{25}$.

(Fig. 1)

\footnotetext{
${ }^{18}$ Pitulko Vladimir Victorovich, Nikolsky Pavel A., Girya E. Yu, Basilyan Alexander E., Tumskoy Vladimir E., Koulakov Sergei A., Astakhov, Sergei N., Pavlova E. Yu, Anisimov Mikhail A. " The Yana RHS Site: Humans in the Arctic Before the Last Glacial Maximum ", Science, 303(5654), 2004, p. 52-56.

${ }^{19}$ Kitchen Andrew, Miyamoto Michael M., Mulligan Connie J., « A Three-Stage Colonization Model for the Peopling of the Americas ", PLoS ONE, 3(2), 2008, p. e1596.

${ }^{20}$ Quintana-MurciLluis, Semino Ornella, Bandelt Hans-J., Passarino Giuseppe, McElreavey Ken, SantachiaraBenerecetti A. Silvana, « Genetic evidence of an early exit of Homo sapiens sapiens from Africa through eastern Africa ", Nature genetics, 23(4), 1999, p. 437-441.

${ }^{21}$ Armitage Simon J., Jasim Sabah A., Marks Anthony E., Parker Adrian G., UsikVitaly. I., Uerpmann Hans-Peter, "The Southern Route "Out of Africa": Evidence for an Early Expansion of Modern Humans into Arabia ", Science, 331(6016), 2011, p. 453-456.

${ }^{22}$ Allen Jim, O'Connell James F., "The long and the short of it: Archaelogical approaches to determining when humans first colonised Australia and New Guinea ", Australian Archaeology, 57(57), 2003, p. 5-10.

${ }^{23}$ Birdsell Joseph B., " The recalibration of a paradigm for the first peopling of Greater Australia ", dans Sunda and Sahul: Prehistoric Studies in Southeast Asia, Melanesia and Australia, éd. par J. Allen, J. Golson, et R. Jones, London, AcademicPress, 1977, p. 113-167.

24 Coupé Christophe, Hombert Jean-Marie, « Les premières traversées maritimes: Une fenêtre sur les cultures et les langues de la préhistoire ", dans Aux origines des langues et du langage, éd. Par J.-M. Hombert, Paris, Fayard, 2005b, p. 118-161.

${ }^{25}$ Coupé C., Hombert J.-M., « Les premières traversées... ", op.cit.
} 


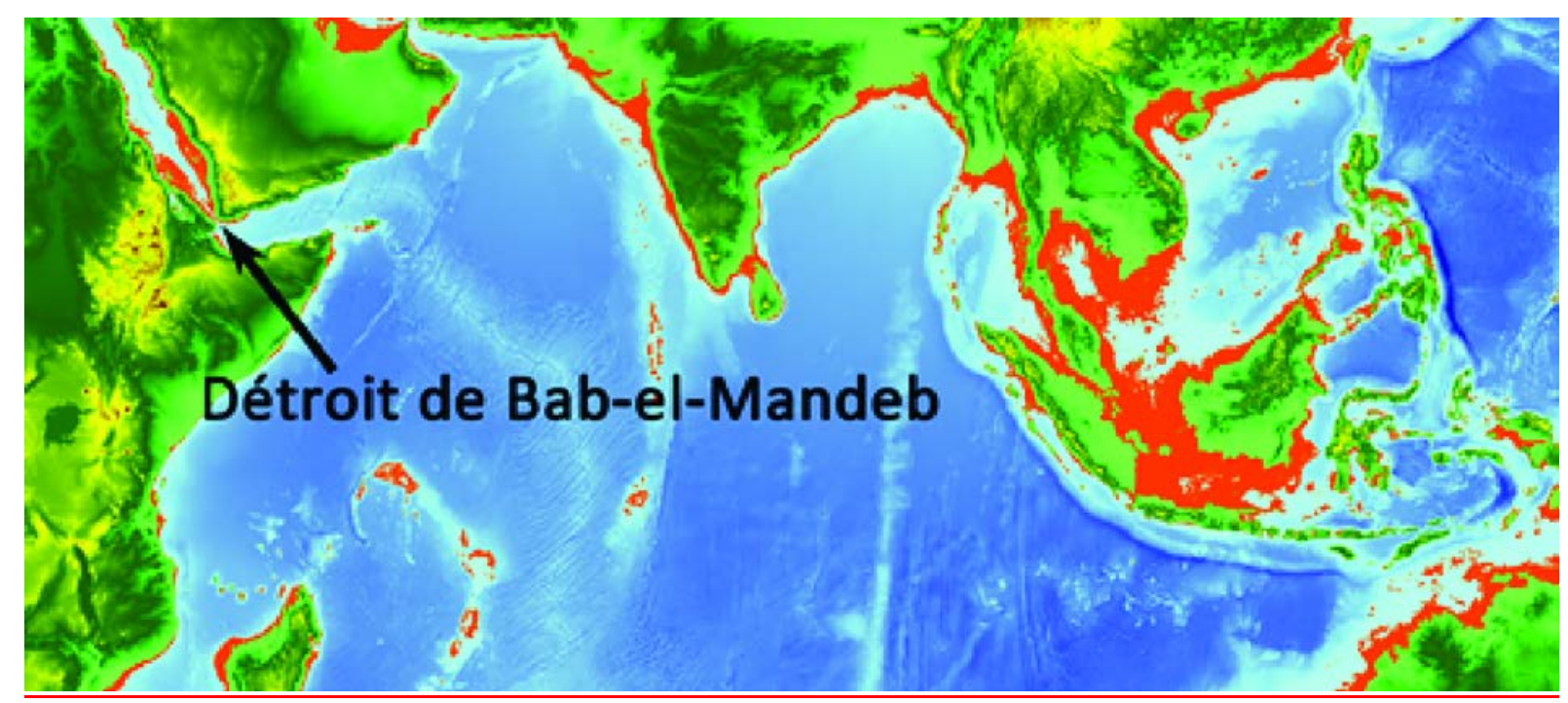

Figure 1 : Différence approximative du niveau des eaux entre -60 000 ans et aujourd'hui. Les zones rouges représentent les terres alors émergées et aujourd'hui immergées. Carte réalisée avec la base de données topographiques Etopo $1^{26}$ et le logiciel QGIS $2.8^{27}$.

\section{Une première grande transition de peuplement}

La sortie hors d'Afrique d'Homo sapiens est une transition majeure dans le peuplement de la Terre. Il est intéressant de mieux la caractériser sur la base des éléments scientifiques précédents, avant d'envisager ce qui a pu en être le moteur.

Deux régimes, l'un précédant la transition et l'autre la suivant, peuvent être définis pour en souligner les points communs et les différences. Le premier correspond à une présence d'Homo sapiens restreinte à l'Afrique, il y a plus de 100000 ans. Le second prend place à la suite des épisodes migratoires qui conduisent à la colonisation de l'Europe, de l'Asie et des Amériques par nos ancêtres. On peut estimer que ce deuxième régime est bien en place il y a environ 15000 ans. La colonisation des îles du Pacifique est beaucoup plus tardive ${ }^{28}$ et peut être laissée à part, de même que les évolutions culturelles qui accompagnent ou découlent de la découverte de l'agriculture: sédentarisation, nouvelles pratiques religieuses etc. La transition qui concerne le passage du premier au second de ces régimes se caractérise ainsi par les différentes vagues migratoires de notre espèce à la surface de la planète, en Afrique et sur les autres continents.

Dans l'analyse des deux régimes pré- et post-transition, nous pouvons d'abord nous pencher sur les « modes d'habiter » et « l'occupation de l'espace » (cf. figure 4, chapitre 2 ).

Les deux régimes sont caractérisés par une structure démographique locale identique : des populations humaines structurées en petits groupes nomades de quelques dizaines d'individus - une trentaine en moyenne ${ }^{29}$. Les densités de population, si elles ont lentement augmenté avec le temps, étaient alors très faibles : la population globale est estimée à plus ou moins 1 million d'individus il y a 40000 ans, et à 6-7 millions d'individus il y a 8000 ans $^{30} 31$.

\footnotetext{
${ }^{26}$ Amante Chris, Eakins Barry W., « ETOPO1 1 Arc-Minute Global Relief Model: Procedures, Data Sources and Analysis ", NOAA Technical Memorandum NESDIS NGDC-24, 2009.

${ }^{27}$ Quantum GIS Development Team. Quantum GIS Geographic Information System. Open Source Geospatial Foundation Project. 2015.

${ }^{28}$ Irwin Geoffrey, The prehistoric exploration and colonisation of the Pacific, Cambridge, Cambridge University Press, 1992.

${ }^{29}$ Hassan Fekri A., Demographic Archaeology, New York, Academic Press, 1981.

${ }^{30}$ Biraben Jean-Noël, « The rising numbers of humankind », Population \& Societies, 394, 2003, p. 1-4.
} 
Localement, de nombreux sites archéologiques suggèrent que les lieux d'habitation de nos ancêtres n'étaient pas choisis au hasard, mais répondaient à différents critères : abris en haut d'un promontoire, offrant une bonne protection contre les prédateurs, à proximité de sources d'eau etc. Les données sont moins précises pour les périodes plus anciennes mais les évolutions qui ont pu prendre place au cours du temps ne sont pas nécessairement directement liées à la transition. À un niveau global ensuite, la différence entre les deux régimes est importante, puisqu'avant la transition, la population humaine est cantonnée à un continent, alors qu'elle en recouvre plusieurs par la suite et en particulier des aires auparavant vierges de toute présence humaine - l'Australie et les Amériques.

Si l'on met en lumière les « formes de mobilité » dans les deux régimes stationnaires, outre le nomadisme déjà mentionné, il est possible de souligner une évolution générale vers une moindre contrainte de l'environnement sur les déplacements. En effet, si les plus anciennes migrations d'Homo sapiens en Afrique respectaient davantage la topographie et le biotope, les routes migratoires le long des côtes du sud de l'Asie puis vers l'Australie à partir du sud-est asiatique suggèrent le franchissement d'obstacles imposants: des régions arides ou au contraire marécageuses, des mangroves et les deltas de grands fleuves ${ }^{32}$, et surtout les vastes étendues d'eau de la région de Wallacea franchies pour gagner le Sahul. Le passage vers les Amériques par le détroit de Béring, plus tardif, est un autre exemple frappant de l'adaptation de nos ancêtres à des environnements difficiles. Ainsi, même si les évolutions du climat ont déterminé en partie les déplacements des hommes ${ }^{33}$, il apparait clairement que ceux-ci se sont affranchis au fur et à mesure de leur niche écologique initiale. Dans le cas particulier de la navigation, une évolution plausible est ainsi le passage de traversées sur de courtes distances, peut-être accidentelles, à des traversées bien plus longues, intentionnelles et planifiées ${ }^{34}$. Il est possible que des motivations alors inédites aient sous-tendu le développement de ces nouvelles formes de mobilité, mais chercher à les identifier précisément reste un exercice spéculatif.

Le regard que nous pouvons porter sur le passé ne nous permet que partiellement d'évaluer les échecs ${ }^{35}$ de certains épisodes migratoires: de nombreuses tentatives de migration ont pu échouer sans laisser de traces et il est difficile de savoir si un territoire a été difficile à « conquérir ». Les discussions autour de la colonisation du Sahul illustrent ce point, avec une opposition entre des scénarios de peuplement accidentels et intentionnels. Dans le premier cas, quelques individus canotant à proximité des côtes des îles de Wallacea auraient été emportés par le vent et les courants jusqu'au Sahul. De tels scénarios accidentels se heurtent toutefois aux faibles chances de survie des êtres humains pris dans une telle mésaventure, en particulier s'ils ne disposaient pas d'eau et de nourriture le temps de la traversée. Ils sont aussi problématiques vis-à-vis du nombre d'individus nécessaires en théorie pour la conquête réussie d'un nouveau territoire ${ }^{36}$. Différents auteurs ${ }^{37}$ leur préfèrent donc des scénarios

\footnotetext{
${ }^{31}$ Bocquet-Appel Jean-Pierre, « When the World 's Population Took Off: The Springboard of the Neolithic Demographic Transition », Science, 333(July), 2011, p. 560-561.

${ }^{32}$ Field Julie S., Petraglia Michael D., Lahr Marta Mirazon, « The southern dispersal hypothesis and the South Asian archaeological record: Examination of dispersal routes through GIS analysis ", Journal of Anthropological Archaeology, 26(1), 2007, p. 88-108.

${ }^{33}$ Stewart John R., Stringer Christopher B., " Human Evolution Out of Africa: The Role of Refugia and Climate Change ", Science, 335(March), 2012, p. 1317-1322.

${ }^{34}$ Coupé C., Hombert J.-M., Les premières traversées ..., op.cit.

${ }^{35}$ Le terme de "échec » est utilisé ici pour indiquer qu'une migration qui a effectivement été réalisée à un moment donné n'a pas donné lieu à un peuplement pérenne. Notons cependant que nous ne connaissons pas les objectifs de ces groupes et c'est par simplification que nous qualifions leurs tentatives en termes de réussite ou d'échec.

${ }^{36}$ McArthur Norma, Saunders I. W., Tweedie R. L., "Small population isolates: A micro-simulation study ", Journal of the Polynesian Society, 85(3), 1976, p. 307-326.
} 
« intentionnels », selon lesquels les traversées n’ont pas été le fruit du hasard, mais ont été planifiées au sein des groupes humains, se sont effectuées à vue, avec des préparatifs renforçant considérablement les chances de survie. Même avec de telles précautions, il n'est pas improbable que de nombreux êtres humains aient perdus la vie dans une telle entreprise, et que la colonisation ait été le résultat de plusieurs traversées réussies étalées au cours du temps.

En ce qui concerne les «manières de se nourrir » et le «rapport plus général à l'environnement », les deux régimes avant et après la transition sont identiques en ce qui concerne une subsistance reposant avant tout sur la chasse et la collecte. Toutefois, lors de la transition, on peut noter des progrès qui dénotent une plus grande compréhension et une meilleure exploitation de l'environnement. Outre l'occupation de différents écosystèmes, des sites archéologiques en Nouvelle-Guinée suggèrent l'abattage de certaines plantes au profit de la croissance d'autres plantes comestibles il y a près de 50000 ans $^{39}$. En Australie, l'évolution du climat local et de la mousson il y a 40000 ans indique un accroissement des feux, probablement utilisés alors pour orienter les proies lors des chasses ${ }^{40}$. L'analyse des restes de chasse et d'alimentation montrent en outre les différences de régime alimentaires selon les saisons, et en particulier la chasse de certains animaux en fonction de leur présence saisonnière et de leur schéma de mobilité ${ }^{41}$. On note également le développement de la pêche ${ }^{42}$, qui a pu être particulièrement significatif pour les groupes qui longèrent les côtés d'Asie en direction de l'Asie du sud-est : la voie de migration côtière, à l'interface entre deux milieux, l'un marin et l'autre terrestre, a pu favoriser le développement des techniques de pêche ${ }^{43}$ ou la collecte de coquillages.

Les "formes de socialité » sont difficiles à identifier. Les interactions entre groupes ont été faibles jusqu'à des époques récentes, même si les contraintes du brassage génétique et la distribution spatiale du matériel lithique suggèrent que différents échanges devaient avoir lieu $^{44}$. Estimer leur fréquence est difficile à cause du manque de données. On peut toutefois estimer un ordre de grandeur grâce à des simulations : la périodicité était plutôt de l'ordre de l'année que du mois ou de la décennie ${ }^{45}$. Ce n'est qu'il y a environ 15000 ou 20000 ans que le registre archéologique contient des traces nettes d’échanges sur de longues distances ou de

\footnotetext{
${ }^{37}$ Bednarik Robert G., « The origins of navigation and language », The Artefact, 1997, 20, p.16.

${ }^{38}$ Coupé C., Hombert J.-M., « Les premières traversées ... », op.cit.

${ }^{39}$ Summerhayes Glenn R., Leavesley Matthew, Fairbairn Andrew, Mandui Herman, Field Judith, Ford Anne, Fullagar Richard, "Human adaptation and plant use in highland New Guinea 49,000 to 44,000 years ago ", Science, 330(6000), 2010, p. 78-81.

40 Johnson Beverly J., Miller Gifford H., Fogel Marilyn L., Magee John W., Gagan M. K., Chivas Allan R., « 65,000 Years of Vegetation Change in Central Australia and the Australian Summer Monsoon ", Science, 284(5417), 1999 , p. 1150-1152.

${ }^{41}$ Niven Laura, Steele Teresa E., Rendu William, Mallye Jean-Baptiste, McPherron Shanon P., Soressi Marie, Jaubert Jacques, Hublin Jean-Jacques, "Neandertal mobility and large-game hunting: The exploitation of reindeer during the Quina Mousterian at Chez-PinaudJonzac (Charente-Maritime, France) ", Journal of Human Evolution, 63(4), 2012, p. 624-635.

${ }^{42}$ Hu Yaowu, Shang Hong, Tong Haowen, Nehlich Olaf, Liu Wu, Zhao Chaohong, Yu Jincheng, Changsui Wang, Trinkaus Erik, Richards Michael P., "Stable isotope dietary analysis of the Tianyuan 1 early modern human ", Proceedings of the National Academy of Sciences of the USA, 106(27), 2009, p. 10971-10974.

${ }^{43}$ O'Connor Sue, Ono Rintaro, Clarkson Chris, " Pelagic Fishing at 42,000 Years Before the Present and the Maritime Skills of Modern Humans ", Science, 334(6059), 2011, p. 1117-1121.

${ }^{44}$ Duke Christopher, Steele James, " Geology and lithic procurement in Upper Palaeolithic Europe: A weightsof-evidence based GIS model of lithic resource potential ", Journal of Archaeological Science, 37(4), 2010 , p. 813-824. Elsevier Ltd.

${ }^{45}$ Coupé Christophe, Hombert Jean-Marie, « Polygenesis of linguistic strategies: a scenario for the emergence of language ", dans Language Acquisition, Change and Emergence: essays in evolutionary linguistics, éd. par Hong J. Minett et W. S.-Y. Wang, Kong, City University of Hong Kong Press, 2005a, p.153-201.
} 
l'existence d'unités culturelles régionales, par exemple au niveau des ornements personnels ${ }^{46}$. Au sein des groupes, il est difficile d'aller au-delà de distinctions générales dans la division du travail, que ce soit entre les sexes ou selon un degré d'expertise. On peut noter toutefois dès le premier régime la présence de traces symboliques sur des outils, ou celle d'objets de parure, qui sont probablement le reflet tangible des relations sociales d'alors ${ }^{47}$. Au cours de la transition, la spécialisation de plus en plus grande des outils suggère des activités toujours plus diversifiées, et probablement le développement d'expertises différenciées chez les membres du groupe. Le fort développement de peintures, de sculptures ou d'autres objets mobiliers à forte valeur symbolique vont sûrement de pair avec une densification des représentations et des activités culturelles: croyance en des entités surnaturelles, rites funéraires ou initiatiques etc.

Pour finir, les formes de pouvoir, que ce soit entre groupes ou au sein des groupes, nous sont inconnues avant des périodes très récentes. Seule la projection de référents ethnographiques modernes, avec par exemple le rôle joué par des individus comme les chamanes, peuvent ici servir de repères ${ }^{48}$.

Il apparaît ainsi, à l'analyse des grandes dimensions qui caractérisent les régimes avant et après la transition, que l'expansion spatiale de notre espèce au cours du temps est un élément clé de la transition. Différents développements accompagnent cette expansion, mais ceux-ci sont graduels et il n'est pas facile de dire s’ils sont spécifiquement la conséquence des migrations (avec par exemple la rencontre d'autres espèces ou d'autres environnements) ou des marques d’un développement plus général et autonome de notre espèce.

\section{Les causes de la première transition}

Les caractéristiques précédemment décrites des régimes pré- et post-transition peuvent être articulées les unes aux autres. Toutefois, il est possible d'aller plus loin en mettant en lumière quelles ont pu être les causes de la transition.

Les évolutions du climat et de l'environnement peuvent expliquer en partie les migrations des populations humaines. En particulier, des conditions plus difficiles ont pu conduire à l'extinction de populations, ou tout du moins à leurs déplacements, avec des phénomènes de contraction géographique et l'abandon de certaines régions trop froides, trop arides etc. Ceci est postulé par exemple pour Homo sapiens au Levant il y a 75000 ans $^{49}$, mais d'autres réponses démographiques à l'environnement sont aussi observées au cours du stade 3 du dernier épisode glaciaire (de 60000 ans à 25000 ans avant aujourd'hui) pour les Hommes modernes et les Hommes de Neandertal ${ }^{50}$. Toutefois, comme déjà souligné, Homo sapiens a développé une plus grande autonomie vis-à-vis de l'environnement, rendue possible grâce à un développement culturel et technique important, et une meilleure compréhension de son fonctionnement. Ces progrès témoignent de l'existence de capacités cognitives accrues par rapport à celles des espèces humaines plus anciennes. On peut voir en elles un, sinon le

\footnotetext{
${ }^{46}$ Vanhaeren Marian, D’Errico Francesco, « Aurignacian ethno-linguistic geography of Europe revealed by personal ornaments ", Journal of Archaeological Science, 33(8), 2006a, p. 1105-1128.

${ }^{47}$ Bouzouggar Abdeljalil, Barton Nick, Vanhaeren Marian, D’Errico Francesco, Collcutt Simon, Higham Tom, Hodge Edward, et al., " 82,000-year-old shell beads from North Africa and implications for the origins of modern human behavior ", Proceedings of the National Academy of Sciences of the United States of America, 104(24), 2007, p. 9964-9.

${ }^{48}$ Lewis-Williams James David, Clottes Jean, "The Mind in the Cave the Cave in the Mind: Altered Consciousness in the Upper Paleolithic ", Anthropology of Consciousness, 9(1), 1998, p. 13-21.

${ }^{49}$ Shea John J., « Transitions or turnovers? Climatically-forced extinctions of Homo sapiens and Neanderthals in the east Mediterranean Levant », Quaternary Science Reviews, 27(23-24), 2008, p. 2253-2270.

${ }^{50}$ Bocquet-Appel Jean-Pierre, Demars Pierre-Yves, « Population Kinetics in the Upper Palaeolithic in Western Europe ", Journal of Archaeological Science, 27(7), 2000b, p. 551-570.
} 
moteur de la transition. Différentes évolutions cognitives ont été mises en avant : développement de la mémoire de travail ${ }^{51}$, fluidité cognitive permettant le transfert de fonctions d'analyse d'un domaine cognitif à un autre ${ }^{52}$ etc. De telles évolutions, en particulier envisagées de concert, peuvent rendre compte de nombre des évolutions observées au cours des 100000 dernières années.

Même si des chercheurs comme Klein ${ }^{53}$ ont proposé dans les années 1990 que des évolutions cognitives majeures aient pris place il y a environ 50000 ans, peu de temps avant l'explosion culturelle du Paléolithique supérieur, l'événement de spéciation qui a donné le jour à Homo sapiens est le meilleur moment pour envisager de telles transformations. Des modifications des structures cérébrales peuvent en effet être la base physiologique de tels changements. Il faut toutefois expliquer alors le décalage entre l'apparition de notre espèce et le caractère tardif de certaines manifestations comportementales, comme les peintures rupestres. Différents éléments sont ici à prendre en compte. Le premier est que notre vision d'Homo sapiens avant sa sortie d'Afrique est encore très parcellaire. Alors que le sol européen, plus densément fouillé et depuis plus longtemps que l'Afrique, nous fournit de nombreux sites pour des périodes récentes, l'accumulation de découvertes en Afrique recule progressivement les débuts de la modernité cognitive et culturelle humaine ${ }^{54}$. Un second point important est que la possession d'aptitudes cognitives ne signifie pas nécessairement leur mise en pratique. Le cas de l'écriture illustre bien la situation : alors que tout être humain peut apprendre à écrire, nombreuses sont les communautés humaines à recourir uniquement à une communication orale. On peut ainsi parler de "potentiel cognitif », et le passage du potentiel à sa réalisation effective dépend de conditions culturelles, sociales et environnementales particulières, comme peut-être la rencontre avec d'autres espèces ou le besoin de faire face à de nouveaux environnements. Le biologique, le cognitif et le culturel sont ainsi liés les uns aux autres d'une façon complexe, avec des interactions pouvant être réciproques. Les causes des migrations humaines hors d'Afrique forment ainsi probablement un complexe difficile à décomposer.

Parmi les acquis cognitifs de notre espèce, le langage dans sa forme moderne occupe une place importante. Les paléoanthropologues s'accordent pour attribuer un langage moderne aux Homo sapiens lors de l'explosion culturelle qui prend place il y a 45000 ans, surtout en Europe, mais la question est plus ouverte pour des époques antérieures. La colonisation de l'Australie il y a un peu plus de 50000 ans, avec la construction d'embarcations robustes et la possible planification des traversées, constitue un autre point d'ancrage intéressant. La maîtrise technique des navigateurs rend compte d'un développement et d'une transmission des savoirs au cours du temps, d'une coopération importante et de l'élaboration d'un projet partagé au sein du groupe, celui de prendre la mer pour gagner une terre distante. Tout ceci ne semble guère possible à l'aide uniquement de signaux rudimentaires liés aux émotions ou à la satisfaction de besoins immédiats. Il semble dès lors qu'une communication essentiellement moderne était alors déjà bien en place. Plus généralement, on peut se demander si le langage moderne a joué un rôle déterminant dans le succès des migrations hors d'Afrique, en soutenant par ses dispositifs expressifs des interactions plus complexes entre les individus.

\footnotetext{
${ }^{51}$ Wynn Thomas, Coolidge Frederick L., « The implications of the working memory model for the evolution of modern cognition », International journal of evolutionary biology, 2011.

${ }^{52}$ Mithen Steven, « From domain specific to generalized intelligence: a cognitive interpretation of the Middle/Upper Palaeolithic transition ", dans The ancient mind. Elements of cognitive archaeology, éd. par C. Renfrew et E. B. W. Zubrow, Cambridge University Press, 1994, p. 29-39.

${ }^{53}$ Klein Richard G., "The Human Career ", Human Biological and Cultural Origins, Chicago et London: The University of Chicago Press, 1999.

${ }^{54}$ Mcbrearty Sally, Brooks Alison S., « The revolution that wasn't: a new interpretation of the origin of modern human behavior ", Journal of human evolution, 39(5), 2000, p. 453-563.
} 
Déterminer les causes de la transition ne permet pas de spécifier complètement les propriétés des migrations qui en sont la signature spatiale. L'expansion géographique et temporelle de notre espèce, si elle découle vraisemblablement de nouvelles capacités cognitives, doit aussi pour être comprise être étudiée selon l'angle des processus d'interaction entre les groupes humains et leur environnement. Le but de la modélisation développée dans ce chapitre est ainsi d'envisager comment a pu se faire la conquête de nouveaux espaces aux ressources hétérogènes et limitées. Le modèle a été développé en deux temps. Dans une première phase, les auteurs "géographes-modélisateurs » se sont inspirés du récit de leurs collègues « linguistes-thématiciens » sur les grandes migrations de Homo sapiens pour développer le modèle HU.M.E (HUman Migrations and Environment). Ce modèle a pour objectif de simuler les modes de peuplement de nouveaux territoires dans un contexte de perturbations environnementales. Il s'agit notamment d'explorer le rôle de mécanismes d'innovation et de diffusion sur la manière dont la colonisation de peuplement évolue sur le long terme. Dans un deuxième temps, ce modèle général a été confronté et adapté à la question spécifique de la sortie d'Afrique d'Homo sapiens décrite dans la première partie de ce chapitre ${ }^{55}$.

\section{Modélisation de la « conquête " de nouveaux territoires}

Plusieurs questions abordées précédemment peuvent être éclairées par une démarche modélisatrice : comment se sont répandus les groupes humains en Afrique et en Asie ? À quelle vitesse ? Quel a été l'impact de l'accumulation progressive des savoirs? Des modèles correspondant à des philosophies différentes permettent d'aborder ces questions et on peut notamment distinguer $\mathrm{s}$ les modèles visant à reproduire une réalité passée et ceux explorant une expérience de pensée ${ }^{56} 57$.

Dans le premier cas, l’objectif est de reconstruire le plus fidèlement possible les phénomènes tels qu'ils se sont effectivement produits, et de tester des hypothèses sur les processus qui en ont été la cause. Dans le cas des migrations de la préhistoire, ceci est d'autant plus difficile que la période concernée est éloignée et/ou que des événements, comme la remontée du niveau des eaux évoquée plus haut, ont détruits les traces archéologiques. Dans certains cas les données génétiques ${ }^{58} 5960$ permettent de pallier cette insuffisance des données archéologiques pour estimer l'étendue et la densité du peuplement en différents lieux et à

\footnotetext{
${ }^{55}$ Une démarche différente a été adoptée dans le chapitre suivant où le modèle a été co-construit par les cinq auteurs, « linguistes-thématiciens » et « géographes-modélisateurs », afin de répondre à une question de colonisation d'un territoire dans un contexte plus spécifique ( $c f$. chapitre 5).

${ }^{56}$ Lake Mark W., "Trends in Archaeological Simulation ", Journal of Archaeological Method and Theory, 21, 2014, p. 258-287.

| ${ }^{57}$ Phan D. (dir), Ontologies et modélisation par SMA en SHS, Hermes-Lavoisier, Londres-Paris, 2014, 558p.

${ }^{58}$ Ammerman Albert J., Cavalli-Sforza Luigi Luca, « The Neolithic Transition and the Genetics of Populations in Europe », Princeton University Press, 1984.

${ }^{59}$ Eriksson Anders, Betti Lia, Friend Andrew D., Lycett Stephen J., Singarayer Joy S., von Cramon-Taubadel Noreen, Valdes Paul J., Balloux François, Manica Andrea, " Late Pleistocene climate change and the global expansion of anatomically modern humans ", Proceedings of the National Academy of Sciences of the USA, 109(40), 2012, p. 16089-16094.

${ }^{60}$ Schneider Stefan, Excoffier Laurent, "Estimation of demographic parameters from the distribution of pairwise differenced when the mutation rates vary among sites: application to human mitochondrial DNA ", Genetics, 153, 1999, p. 1079-1089.
} 
différentes époques. Le modèle emblématique d'Ammerman et Cavalli-Sforza ${ }^{61}$ sur la croissance et la dispersion des populations dans les temps anciens, modèle dit de " réactiondiffusion ", a donné lieu à une importante littérature et a été appliqué à de nombreux cas : diffusion du Néolithique en Europe ${ }^{62}$ et en Amérique, migrations des chasseurs-collecteurs au Paléolithique etc.

Les modèles de la seconde catégorie sont plus exploratoires, voire expérimentaux. Il ne s’agit plus de reproduire des faits empiriques mais plutôt d'explorer, avec le modèle et souvent par la simulation, une expérience de pensée. Premo ${ }^{63}$ défend ainsi l'idée qu'un modèle à base d'agents est un outil pour construire des histoires alternatives en multipliant des scénarios de type " what if ». Il propose de remplacer la question : "What happened in region X during period Y ? ", par la suivante: "How likely is it that behavior Q or trait $\mathrm{Z}$ would evolve in the population in region $\mathrm{X}$ during period $\mathrm{Y}$ given a wide range of plausible environmental conditions and alternative histories ? ". De tels modèles sont féconds et permettent de réfléchir, même quand les données sont pauvres, au fonctionnement de différents processus et à la plausibilité de différents changements. Les simulations servent alors à " générer » des hypothèses plutôt qu'à les «tester » comme dans la première catégorie ${ }^{64}$, et à explorer différentes alternatives plausibles d'évolution ${ }^{65}$. Le modèle HU.M.E. s'inscrit dans cette approche.

\section{Modèles de migration et de colonisation de nouveaux espaces : un bref état de l'art}

La transition modélisée par HU.M.E. se rapporte au processus qui conduit un espace initialement vierge de toute présence humaine à se peupler sous l'effet d'un flux migratoire. Plus précisément, le modèle explore des histoires possibles de la colonisation humaine d'un espace inoccupé dans un contexte de perturbations environnementales. L’objectif est d'explorer les effets de différents paramètres et de différentes situations initiales sur la configuration spatiale et le rythme du peuplement. Le processus central est celui de la migration et nous proposons un bref état de la littérature sur la modélisation de celui-ci dans les temps préhistoriques.

Les modèles se différencient d'abord par leur niveau d'abstraction de l'espace géographique. Young ${ }^{66}$ considère un espace abstrait, homogène et isotrope, où se déplacent des agents qui représentent des individus ou des groupes (ce modèle est exposé dans le chapitre 3). De nombreux travaux ancrent au contraire leur modèle dans une géographie réaliste: par exemple, pour modéliser la diffusion du Néolithique dans l'espace européen, Parisi et al. ${ }^{67}$ reconstituent les potentiels agricoles de cette région à une échelle fine (cellules de $70 \mathrm{~km}^{2}$ ). Le niveau d'abstraction concerne par ailleurs les mécanismes modélisés. Les modèles les plus abstraits représentent le déplacement comme une simple marche aléatoire (chapitre 3). À

\footnotetext{
${ }^{61}$ Ammerman A. J., Cavalli-Sforza L. L., The Neolithic Transition... op. cit.

${ }^{62}$ Fort Joaquim, « Demic and cultural diffusion propagated the Neolithic transition across different regions of Europe ", Journal of The Royal Society Interface, 12(106), 20150166, 2015.

${ }^{63}$ Premo Luke S., " Exploratory Agent-based Models: Towards an Experimental Ethnoarchaeology », dans Digital Discovery: Exploring New Frontiers in Human Heritage, éd. par J. T. Clark et E. M. Hagemeister, CAA, 2006, Computer Applications and Quantitative Methods in Archaeology, Proceedings of the 34th Conference, Fargo, USA, April 2006, Budapest, Archaeolingua, 2007, p. 29-36.

${ }^{64}$ Lake M. W.,Trends in Archaeological..., op.cit.

${ }^{65}$ Chevrier Benoît, " Ni espace ni temps en Préhistoire ancienne: "Out of Africa" ou le paradigm de la flèche ", Mappemonde, 2012, p. 106.

${ }^{66}$ Young David A., "A new space-time computer simulation method for human migration ", American Anthropologist, 104(1), 2002, p. 138-158.

${ }^{67}$ Parisi Domenico, Antinucci Francesco, Natale Francesco, Cecconi Federico, « Simulating the expansion of farming and the differentiation of European languages ", Evolution of Languages, 2008, p. 1-41.
} 
l'opposé, d'autres modèles formalisent explicitement les mécanismes conduisant un individu ou un groupe à se déplacer selon des décisions que l'on peut qualifier de rationnelles. Dans Parisi et al. ${ }^{68}$ par exemple, les agents se déplacent quand la « carrying capacity » de la cellule qu'ils occupent devient insuffisante, c'est-à-dire quand leurs besoins dépassent les ressources disponibles. Au-delà d'un certain nombre d'agents dans la cellule, une partie d'entre eux migre vers une cellule adjacente, choisie au hasard parmi les cellules inoccupées et présentant un potentiel agricole non nul.

Les modèles les plus abstraits sont les plus génériques: en variant un petit nombre de paramètres, un même modèle peut reproduire les grandes lignes d'un processus de colonisation dans des espaces bien distincts et à des périodes différentes. Par exemple, dans le modèle de Young, certaines combinaisons du taux de croissance démographique et de la propension migratoire mènent à un front de colonisation, tel qu'observé au Néolithique en Europe alors que d'autres aboutissent au contraire à une colonisation diffuse de l'ensemble de l'espace, similaire à la colonisation de l'Australie au Pléistocène ${ }^{69}$. D'autres modèles s'inscrivent dans une perspective plus réaliste, que ce soit par l'introduction de données environnementales pour l'espace considéré ${ }^{70}{ }^{71}$, ou de comportements plus sophistiqués des agents $^{72}$.

Le modèle HU.M.E. a été développé avec un système multi-agents (SMA) dans lequel les groupes de chasseurs-collecteurs sont formalisés comme des « agents » (cf. chapitre 3).

\section{Les principales composantes du modèle HU.M.E.}

Le modèle HU.M.E étudie, au moyen de simulations, la colonisation d'un espace inoccupé par des groupes de chasseurs-collecteurs ( $c f$. encadré 1). Il est d'un niveau d'abstraction intermédiaire, avec des règles de comportement très stylisées, un espace stylisé également mais hétérogène. Les déplacements des groupes sont essentiellement motivés par la recherche de ressources. Ils exploitent leur environnement avec un certain savoir-faire (formalisé dans le modèle sous forme d'un niveau technique) qui peut évoluer et garantir une exploitation plus performante. Le but du modèle est d'interroger les rôles respectifs de trois facteurs en interaction - la démographie, la ressource et l'innovation technique - sur la forme, la vitesse et la pérennité de la colonisation d'un nouvel espace.

Deux types d'entités sont définies (figure 2):

- l'agent-groupe, représentant un groupe de chasseurs-collecteurs d'une trentaine d'individus. Il exploite les ressources de son environnement (i.e. de la cellule qu'il occupe) en fonction de son niveau technique et emmagasine de l'énergie. Il est capable d'innover pour exploiter plus efficacement la ressource et d'imiter des groupes voisins pourvus de techniques d'exploitation plus élaborées. Dépourvu de capacité d'anticipation, il se déplace aléatoirement.

- la cellule, correspondant à une portion de territoire. Les cellules exploitables sont caractérisées par un niveau de ressource, qui peut être assimilé à une biomasse. Celle-ci se régénère avec le temps et peut être affectée par des perturbations exogènes.

\footnotetext{
${ }^{68}$ Parisi D., et. al., " Simulating the...», op.cit.

${ }^{69}$ Bowdler Sandra, «The coastal colonization of Australia », dans Sunda and Sahul: Prehistoric Studies in Southeast Asia, Melanesia and Australia, éd. par J. Allen, J. Golson, et R. Jones, London, Academic Press, 1977, p. 205-246.

${ }^{70}$ Heckbert Scott, "MayaSim: An agent-based model of the ancient Maya social-ecological system ", Journal of Artificial Societies and Social Simulation, 16(4), 2013, p. 11.

${ }^{71}$ Parisi D. et. al., "Simulating the...», op.cit.

${ }^{72}$ Barton Michael C., Riel-Salvatore Julien, « Agents of change: modeling biocultural evolution in Upper Pleistocene western Eurasia », Advances in Complex Systems, 15(1-2), 2012.
} 
(Fig. 2)

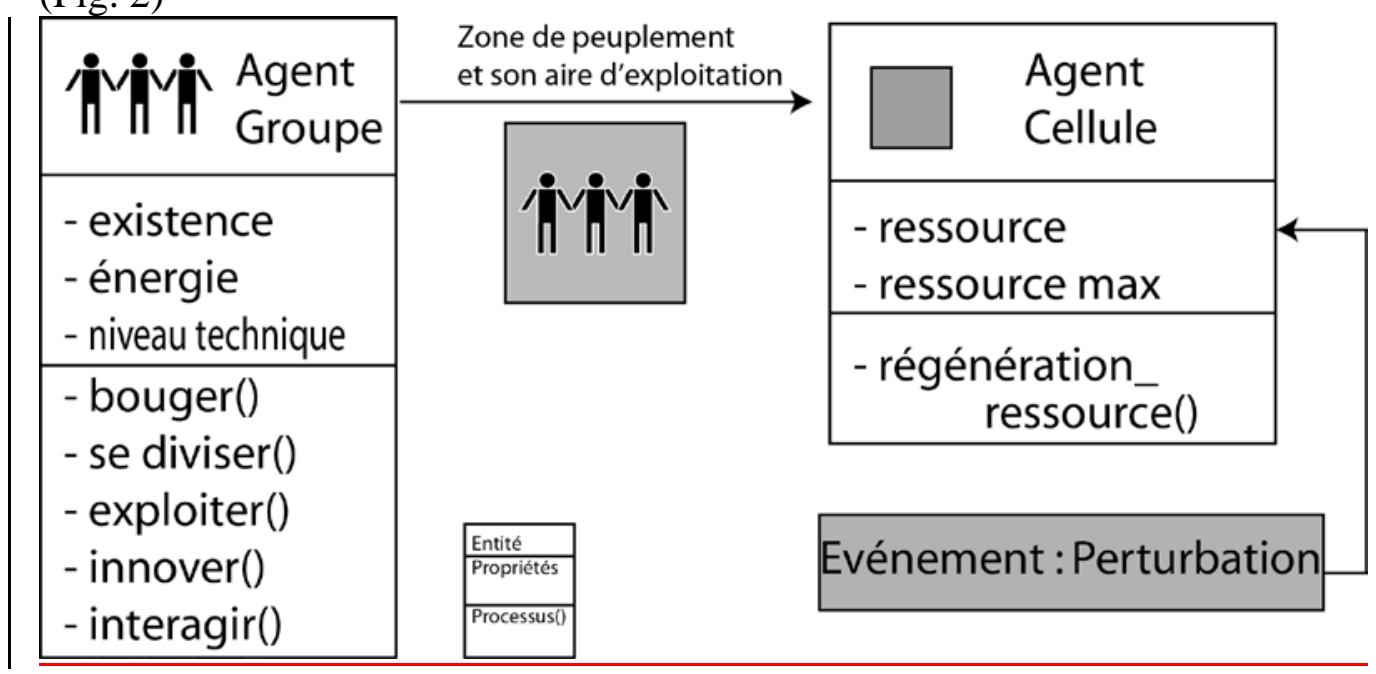

Figure 2 : Les entités, propriétés, relations et processus du modèle.

Deux types d'interactions sont associés à ces entités et jouent un rôle moteur dans l'évolution du système :

- entre l'agent-groupe et son environnement: quand les ressources y sont insuffisantes l'agent-groupe quitte la cellule ;

- entre les différents agents-groupes situés sur la même cellule : relations de compétition pour les ressources et d’imitation, avec la possibilité de transfert du niveau technique. 


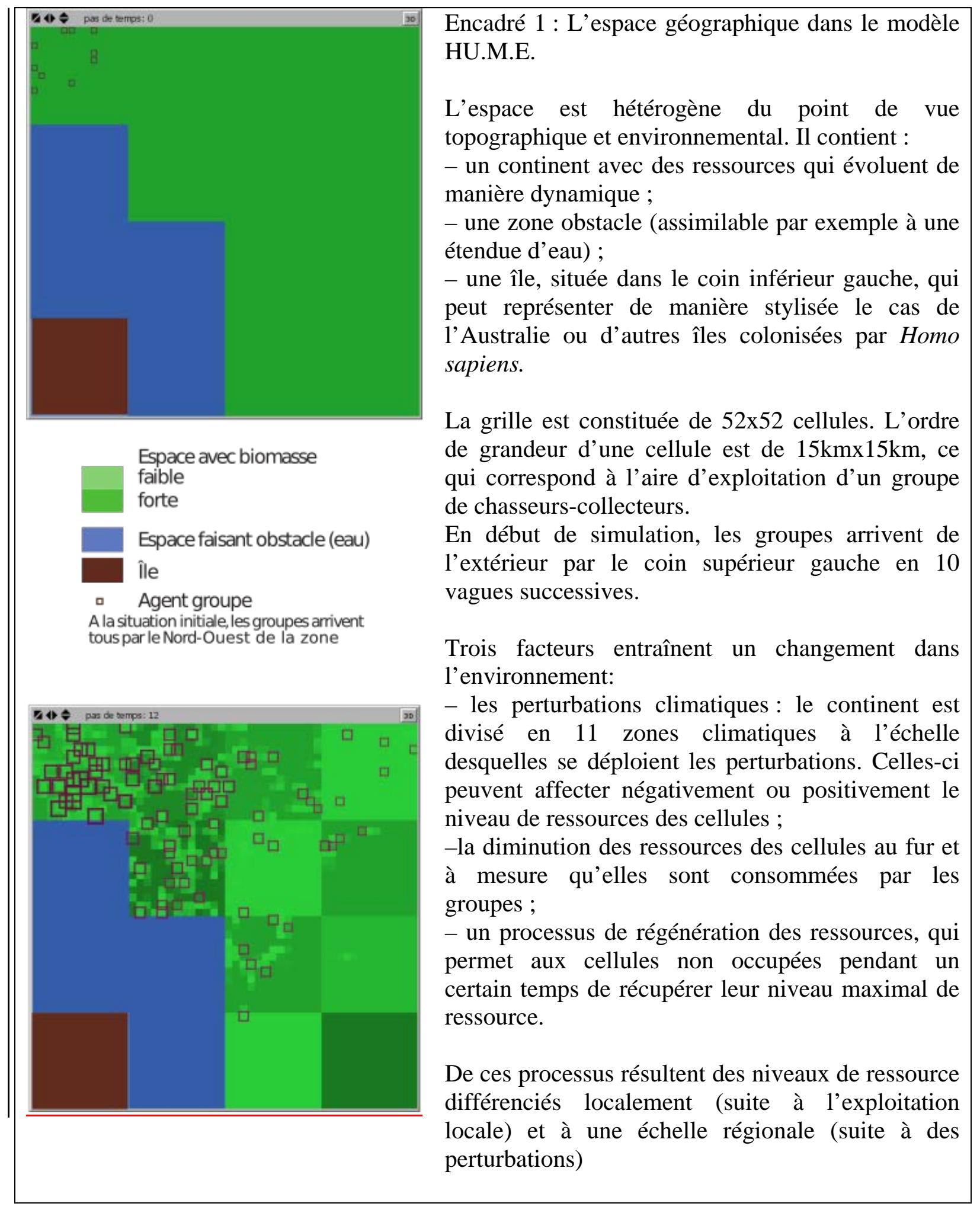

\section{Construction du modèle par blocs successifs}

La présentation du fonctionnement d'un modèle peut prendre différentes formes. Nous proposons ici une approche incrémentale pour introduire les mécanismes génériques du modèle et mettre en évidence le rôle de chacun. Cette construction progressive aboutit à 
quatre versions du modèle (notées V0 à V3), de la plus simple (figure 3.a) à la plus complète (figure 3.b).

(Fig. 3)
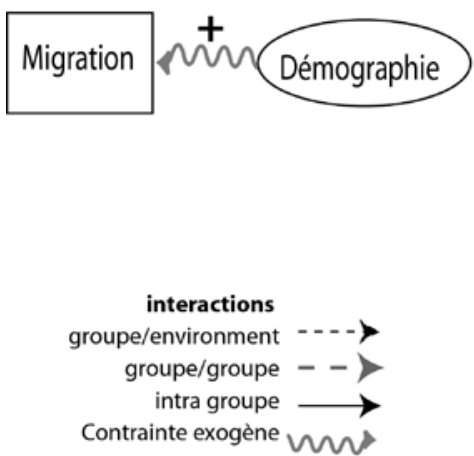

(a)

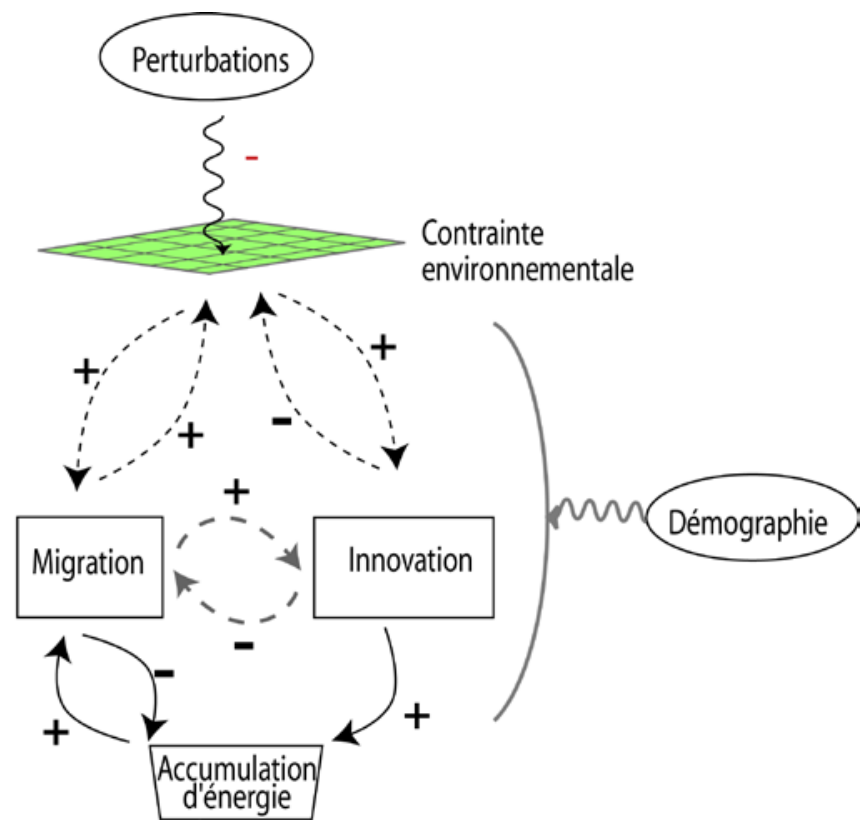

(b)

Figure 3: Représentation schématique du fonctionnement du modèle HU.M.E., dans sa version la plus simplifiée (a) à la plus complète (b).

Dans la version «V0 » du modèle existent seulement deux mécanismes, la migration et la démographie (figure 3.a). La migration se fait d'une cellule à l'autre suivant une marche aléatoire : les agents n’ont ni connaissances ni préférences. L’environnement est neutre: les agents n'ont pas de « raison » de se déplacer, la décision de migrer est simplement contrôlée par un paramètre fixant la probabilité de se déplacer à chaque itération. La croissance démographique est aussi déterminée par un paramètre unique qui donne la probabilité à chaque itération qu'un groupe se scinde en deux et donne ainsi naissance à un nouvel agentgroupe. Avec ces deux seuls mécanismes, le modèle reproduit les deux formes de colonisation observée par Young: i) avec une forte démographie et une faible mobilité, un front de colonisation (figure 4.a) ; ii) avec une faible natalité et une forte mobilité, une colonisation dispersée (figure 4.b).

La deuxième étape (version V1) consiste à introduire des mécanismes utilisant la notion de « ressources ». Ces ressources sont d'une part «externes », extraites de l'environnement, dont l'exploitation sert à la survie des agents-groupes (alimentation, outils). Les ressources peuvent aussi être «internes » et résulter d'un processus d'accumulation des bénéfices apportés par une période de sédentarité: repos, possibilités de préparatifs et de manière générale développement d'un bon état physique et relationnel, etc. De manière métaphorique, ces «ressources internes" ont été formalisées dans le modèle par une variable qualifiée d' " énergie ». L'énergie augmente à chaque itération où le groupe reste sur une même cellule et diminue lors de chaque déplacement. Cette variable intervient ainsi dans les règles formalisant les déplacements des agents-groupes (le déplacement impliquant une dépense en énergie). Elle sert aussi à réguler la démographie, une valeur trop faible entraînant la disparition du groupe. Dans cette version, les contraintes liées aux interactions avec l'environnement (rareté de la ressource), à l'énergie et à la compétition entre les groupes sont 
les moteurs de la migration. Des déplacements « sans raison » sont cependant maintenus, avec une faible probabilité, pour tenir compte d'autres logiques que la réponse aux contraintes de l'environnement ( $c f$. chapitre 6 pour une approche plus détaillée de ces logiques alternatives).

La troisième version (version V2) du modèle intègre les mécanismes d'innovation et d'imitation qui rendent compte de comportements plus élaborés des agents-groupes. L'innovation permet à un groupe d'acquérir un niveau technique plus élevé, qui lui permet d'exploiter plus efficacement la ressource. Si les ressources de la cellule sur laquelle il se trouve sont insuffisantes, un groupe peut répondre à cette pression par une innovation. Grâce à cette innovation, il pourra subvenir à ses besoins sans ressources supplémentaires ${ }^{73}$. L'innovation constitue ainsi une alternative à la migration, avec comme conséquence une augmentation de l'énergie permise par une plus longue période de sédentarité. Un groupe peut aussi innover par imitation : lorsque deux groupes se trouvent sur une même cellule, le groupe de niveau technologique plus faible peut acquérir, par imitation, le niveau de l'autre groupe. Sur la figure 4c qui correspond à cette version V2 du modèle, les groupes sont représentés par des carrés dont la taille est proportionnelle au niveau technique. Une différenciation des groupes émerge rapidement et les niveaux techniques les plus élevés apparaissent dans les zones où la concentration est la plus forte, i.e. celles qui sont les plus proches des premiers lieux colonisés.

Enfin, dans la version la plus complète du modèle, V3, des perturbations aléatoires viennent affecter les ressources. La figure $4 d$ montre comment une perturbation a affecté le niveau de ressource d'une zone centrale de l'espace, créant un obstacle dans le cheminement migratoire des groupes. Seuls ceux ayant accumulé suffisamment d'énergie parviendront à traverser cette bande de terre « inhospitalière ».

Tous ces mécanismes interagissent et conduisent à des situations variées. De plus l'ensemble des comportements sont soumis à de l'aléa, de sorte que n'émerge pas de configuration déterministe. Cette version V3 du modèle est utilisée dans les explorations présentées dans la prochaine section.

(Fig. 4)

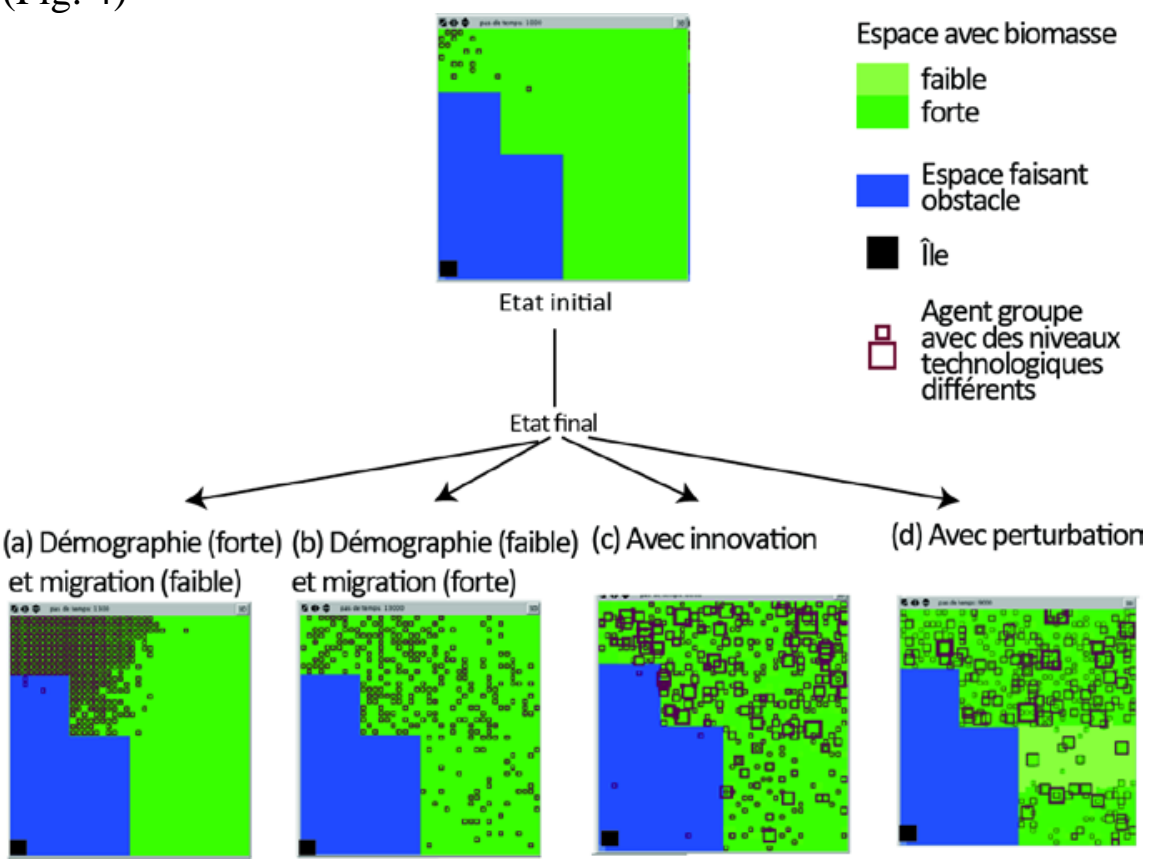

Figure 4 : Dynamiques de colonisation avec différents paramétrages du modèle

\footnotetext{
${ }^{73}$ Autrement dit, le groupe parvient à extraire plus d'énergie de la même quantité de ressources.
} 


\section{Exploration par simulation des conditions de réussite de la colonisation d'un nouvel espace}

Le modèle a été utilisé pour explorer les conditions de réussite versus échec de la colonisation d'un nouvel espace. Il s'agit d'évaluer le type de situation à laquelle conduit chaque simulation et de comparer entre elles différentes simulations en termes de succès ou d'échec. Pour ce faire, trois indicateurs caractérisant l'état du système de peuplement à chaque itération $t$ ont été construits :

- la survie du peuplement, mesurée par le nombre total d'agents-groupes : plus ceux-ci sont nombreux, plus la colonisation est considérée comme une réussite ;

- l'étendue de l'espace colonisé, mesurée d'une part par la distance moyenne des agentsgroupes au point de départ de la colonisation (figure 5.a) et d'autre part par le nombre de cellules occupées. Elle est une deuxième dimension de la réussite de la colonisation;

- la capacité à traverser la mer, mesurée par le pourcentage de groupes ayant atteint l'île (figure 5.a). La discussion menée plus haut sur la colonisation du Sahul mettait en évidence la difficulté de telles traversées. La réussite de ces dernières, avec l'ouverture sur de nouveaux territoires qu'elle apporte, contribue au succès de la colonisation,

L'exploration des sorties des simulations a été réalisée à partir de la plateforme de visualisation « VisuAgents ${ }^{74}$. La figure 5.b représente par exemple le cas d'une colonisation réussie et l'on peut suivre dans le temps de la simulation l'évolution du nombre d'agentsgroupes en fonction de la distance moyenne au point de départ. L'intensité de la couleur du point rend compte de sa position par rapport à la durée de la simulation : en clair sont représentés les états du système au début de la simulation, en foncé les états finaux. Le nombre de groupes commence par diminuer alors que la distance au point de départ augmente rapidement, puis on observe un point de retournement : le nombre d'agents-groupes se met à augmenter au même rythme que la distance au point de départ. Enfin, après environ 400 itérations, cette dernière se stabilise. Le peuplement couvre alors l'ensemble de l'espace.

La mobilisation de ces indicateurs lors de la situation finale (fixée au temps $t=20000)^{75}$ permet de caractériser l'état du système et d'évaluer le degré de réussite de la colonisation de l'espace.

(Fig. 5)

\footnotetext{
${ }^{74}$ Cura Robin, Boukhechba Mehdi, Mathian Hélène, Le Néchet Florent, Sanders Lena, "VisuAgent - Un environnement d'exploration visuelle de données spatio-temporelles issues de simulation ", SAGEO, Grenoble, 2014.

${ }^{75} t=20000$ a été déterminé par expérimentation, c'est le seuil à partir duquel le nombre de groupes n'évolue plus.
} 


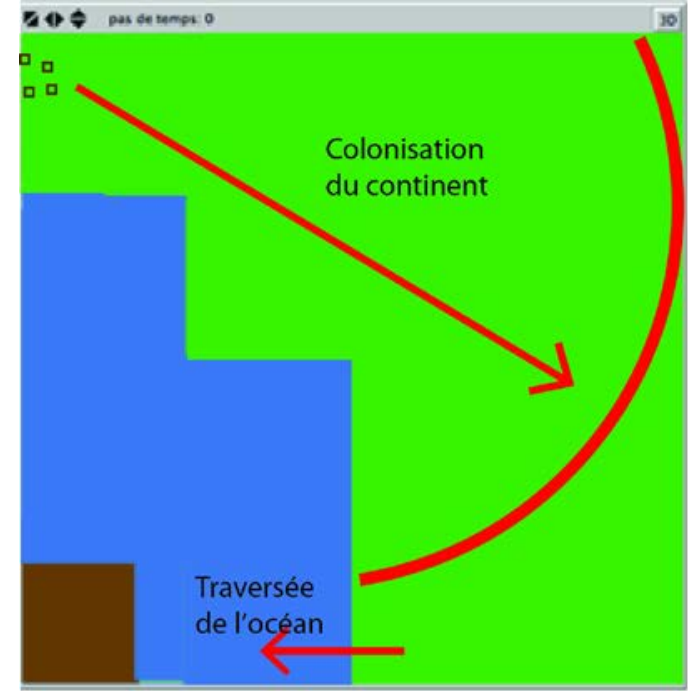

(a) Schématisation du succès d'une colonisation : survie, colonisation et traversée de l'océan

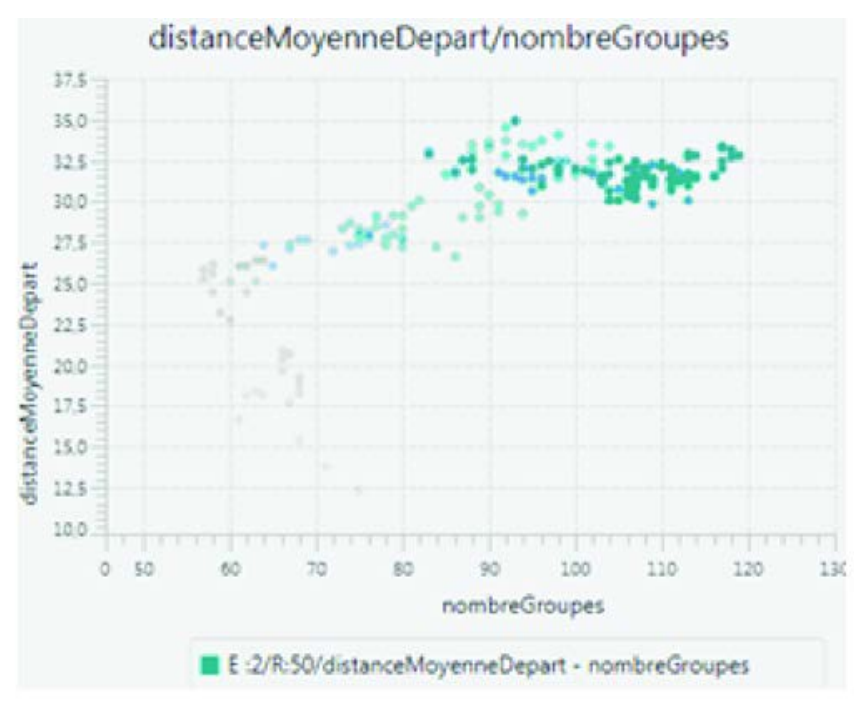

(b) Sorties d'une simulation dans un cas de colonisation réussie : évolution dans le temps (intensité de bleu) de la distance moyenne au centre (ordonnée) par rapport au nombre de groupes (abscisse)

Figure 5 : Mesurer le succès d’une colonisation.

Un plan d'expérience a été conçu afin d'explorer les résultats des simulations et tester leur sensibilité à la variation de quatre paramètres associés aux mécanismes clés du modèle. Il s’agit de la probabilité qu'un groupe se divise (démographie), la quantité d'énergie accumulée en cas de sédentarité, le coût en énergie d'une migration et la probabilité d'innover. Il s'agit d'explorer l'importance relative des différents processus impliqués à l'aide des indicateurs de sortie définis plus haut. Le tableau 1 présente les paramètres impliqués avec leurs domaines de variabilité respectifs.

\begin{tabular}{|l|l|l|l|l|}
\hline Mécanismes & $\begin{array}{l}\text { Paramètres } \\
\text { clés }\end{array}$ & Nom détaillé & Explication & $\begin{array}{l}\text { Valeur de référence } \\
\text { et } \\
\text { domaine } \\
\text { valeur }\end{array}$ \\
\hline Démographie & PR & $\begin{array}{l}\text { Probabilité de } \\
\text { division }\end{array}$ & $\begin{array}{l}\text { Reproduction des groupes } \\
\text { (croissance démographique) }\end{array}$ & $\begin{array}{l}2.10^{-5}\left(2 \text { à } 16.10^{-5},\right. \\
\left.\text { pas de } 2.10^{-5}\right)\end{array}$ \\
\hline Migration & ED & $\begin{array}{l}\text { Energie liée au } \\
\text { déplacement }\end{array}$ & $\begin{array}{l}\text { Dépense d'énergie induite par une } \\
\text { migration }\end{array}$ & $\begin{array}{l}0,12 \text { (0,10 à } 0,20, \\
\text { pas de } 0,02)\end{array}$ \\
\hline Innovation & PI & $\begin{array}{l}\text { Probabilité } \\
\text { d'innovation }\end{array}$ & $\begin{array}{l}\text { Probabilité pour un groupe } \\
\text { d'augmenter son niveau technique }\end{array}$ & $\begin{array}{l}0,055(0,03 \text { à } 0,06, \\
\text { pas de } 0,005)\end{array}$ \\
\hline $\begin{array}{l}\text { Accumulation } \\
\text { d'énergie }\end{array}$ & EA & $\begin{array}{l}\text { Energie } \\
\text { accumulée }\end{array}$ & $\begin{array}{l}\text { Quantité d'énergie accumulée par } \\
\text { pas de temps lorsqu'un groupe reste } \\
\text { sur une cellule }\end{array}$ & $\begin{array}{l}1(0.5 \text { à } 1.5, \text { pas de } \\
0.25)\end{array}$ \\
\hline
\end{tabular}

Tableau 1: Mécanismes et paramètres clés du modèle (dans la $4^{\mathrm{e}}$ colonne sont donnés successivement la valeur dans la simulation dite de référence, les valeurs minimale et maximale du domaine de variation du paramètre, et le pas de variation utilisé).

Afin d'illustrer les résultats des simulations, nous présentons des sorties pour les huit expérimentations correspondant aux variations du premier paramètre, la probabilité qu'un groupe se divise ( $\mathrm{PR}$, dans le tableau 1). Ces expérimentations sont notées de E1 ( $\mathrm{PR}=2.10^{-5}$ ) à E8 $\left(\mathrm{PR}=16.10^{-5}\right)$ et permettent d'explorer la variabilité des résultats toutes choses égales quant aux valeurs des autres paramètres. Les simulations couvrent 20000 pas de temps, avec un relevé tous les 100 pas de temps. Ces relevés permettent de représenter différentes trajectoires d'un indicateur ou d'un croisement d'indicateurs et nous en donnons quelques exemples à titre illustratif. 
La figure 6 croise ainsi, pour l'expérimentation E3, la distance totale parcourue en moyenne par les groupes (en abscisse) avec l'écart-type de cet indicateur (en ordonnée). Ce dernier informe sur le degré d'étalement du peuplement (cas d'une valeur élevée) ou au contraire de concentration de l'ensemble des groupes dans l'espace (cas d'une valeur faible). Ce graphique permet ainsi de suivre la dynamique de la colonisation et de son inscription spatiale. Le nuage de points traduit la trajectoire du système de peuplement, avec une distance totale parcourue qui s'accroit au cours de la simulation. La position sur l'axe des ordonnées rend compte de phases de contraction (faible écart-type des distances au point de départ) et de dispersion des groupes (fort écart-type). On observe que ces phases sont cycliques. Cela est lié aux perturbations qui, en diminuant les ressources sur une partie des cellules, contraignent les groupes à se rapprocher.

(Fig. 6)

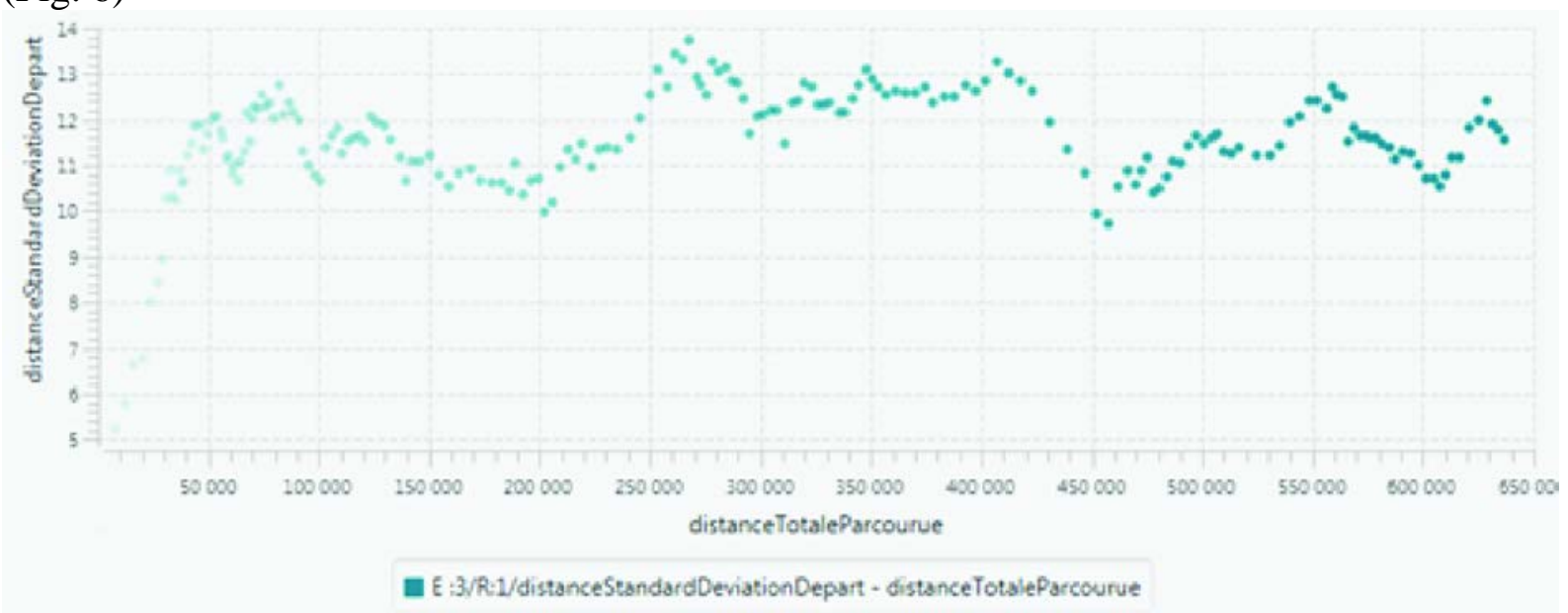

Figure 6 : Suivi de la dynamique de l'expérimentation E3 à travers deux indicateurs évoluant dans le temps, la distance moyenne parcourue et l'étalement du peuplement.

La figure 7 illustre la différence entre les expérimentations E2 (faible valeur de PR, en a) et E7 (forte valeur de PR, en b). Ces figures croisent le nombre de cellules occupées (en ordonnée) avec le niveau technique moyen des groupes (en abscisse). Ainsi, pour une valeur de PR faible, la colonisation est deux fois plus diffuse que pour une valeur élevée (le nombre de cellules occupées atteint 140 pour (a) et seulement 70 pour (b)), et le niveau technologique moyen est en moyenne plus élevé. Ce résultat est en partie lié au fait qu'à une plus forte densité de groupes est associée une meilleure diffusion des niveaux technologiques. On observe dans les deux cas (par l'intensité de couleur) que ce niveau technique suit des cycles au cours du temps. Ces variations sont liées aux perturbations qui conduisent à une rétraction du peuplement sur les cellules les plus riches, ce qui favorise une augmentation de la diffusion des niveaux technologiques.

(Fig. 7) 


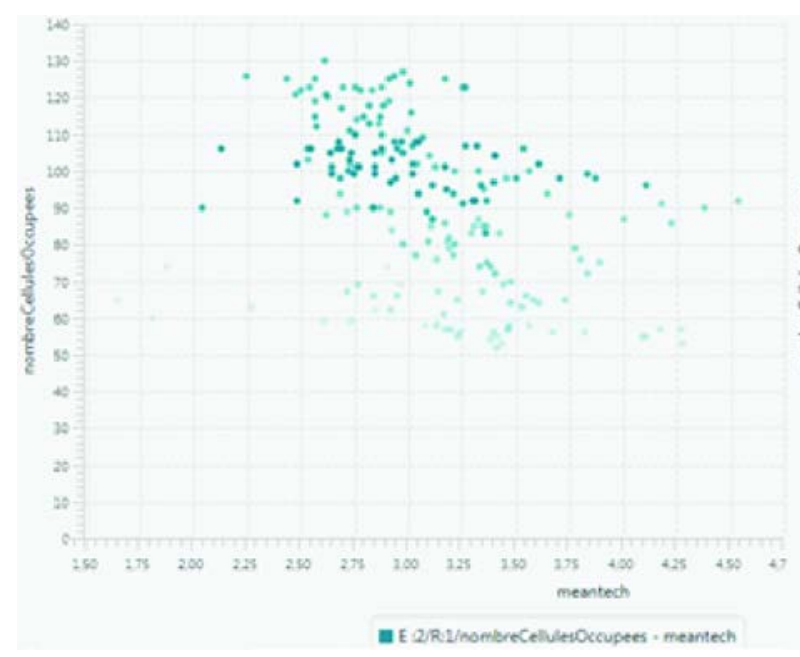

(a)

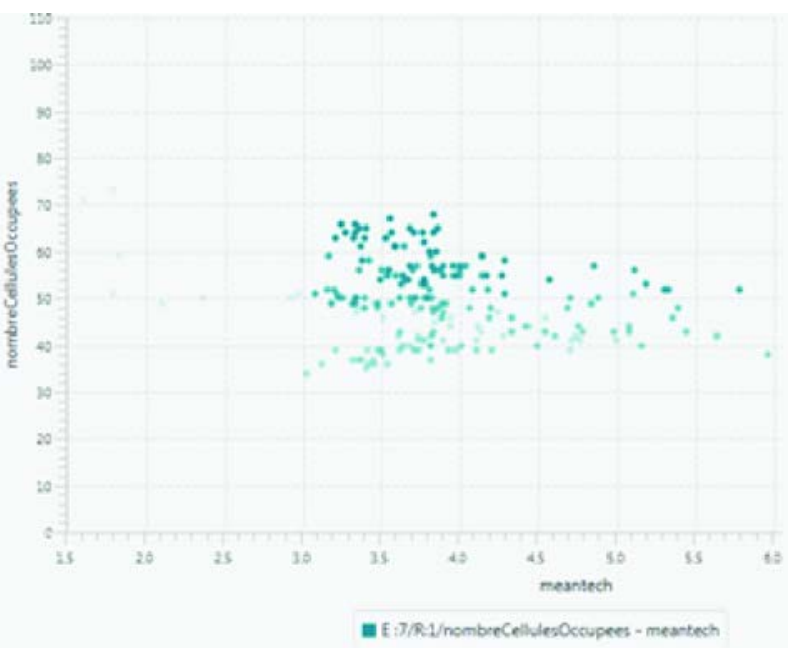

(b)

Figure 7: Diffusion du peuplement et niveau technologique : effets d'un changement de valeur du paramètre PR traduisant la croissance démographique (faible valeur en (a), forte en (b))

Les résultats précédents résultent de simulations utilisant la même graine aléatoire, afin de faire ressortir l'effet d'un changement de valeur d'un paramètre. Le modèle étant stochastique (les actions des agents-groupes sont déterminées par des probabilités), il est cependant intéressant de faire varier cette graine aléatoire et de recourir à plusieurs réplications (300 dans notre cas) pour explorer plus systématiquement les résultats des simulations en termes de réussite ou d'échec de la colonisation. Une comparaison a ainsi été faite entre des expérimentations correspondant aux huit valeurs de taux de reproduction testées. Le tableau 2 montre que la relation entre l'augmentation de ce paramètre et le nombre total de groupes en fin de simulation n'est pas aussi simple que l'on croirait intuitivement. La relation est effectivement régulière pour les expérimentations E1, E2 et E3, le nombre de groupes en fin de simulation augmentant avec le taux de reproduction. Cette régularité est ensuite brisée et les expérimentations E5 et E6 conduisent aux plus faibles valeurs. Les colonisations les plus réussies sont ainsi associées à des taux de reproduction plutôt faibles. Ces résultats laissent supposer une interaction (non introduite explicitement dans le modèle) entre la compétition pour les ressources et la démographie. En effet, un nombre de groupes plus important induit à la fois une meilleure diffusion de l'innovation et une plus forte pression sur les ressources. Il apparait ainsi que pour E5 et E6 les avantages de la croissance sont annulés par le manque de ressources qui rend le système vulnérable. Dans cette situation, une perturbation peut déboucher sur l'extinction d'un nombre important de groupes (E5 et E6) et il faut une forte démographie pour contrecarrer ce phénomène (E7 et E8).

\begin{tabular}{|l|l|l|l|l|l|l|l|l|}
\hline & E1 & E2 & E3 & E4 & E5 & E6 & E7 & E8 \\
\hline PR & $2.10^{-5}$ & $4.10^{-5}$ & $6.10^{-5}$ & $8.10^{-5}$ & $10.10^{-5}$ & $12.10^{-5}$ & $14.10^{-5}$ & $16.10^{-5}$ \\
\hline Nb. & 100 & 120 & 140 & 90 & 10 & 25 & 40 & 70 \\
\hline
\end{tabular}

Tableau 2 : Nombre de groupes (Nb.) en fin de simulation pour huit valeurs du paramètre PR (probabilité de reproduction du groupe)

Une exploration systématique de l'espace des paramètres a montré que certains ensembles de paramètres menaient l'ensemble des 300 réplications au même résultat, certaines aboutissant à l'émergence d'un peuplement durable et couvrant l'ensemble de l'espace alors que d'autres débouchent systématiquement sur l'extinction de l'ensemble des groupes. Nous avons rejeté ces ensembles de paramètres comme non vraisemblables. En effet, même si la situation est 
encore imprécise, nous savons qu'Homo sapiens a réussi à coloniser la quasi-totalité de la planète, tout en connaissant des difficultés et/ou des échecs à certaines époques : disparition du Levant il y a 75000 ans, conquête probablement difficile de l'Australie, difficulté du passage par la Béringie ( $c f$. la première section de ce chapitre).

Le choix a donc été fait de s'intéresser aux ensembles de paramètres conduisant à une certaine variabilité des résultats, c'est-à-dire laissant sa juste place à l'aléa. Certains jeux de paramètres conduisent ainsi, suivant les réplications, soit à un échec, soit à une colonisation totale de la région avec, en plus, certains groupes qui parviennent à traverser l'obstacle que représente la mer. La classification des 300 trajectoires simulées avec le jeu de paramètres choisi comme référence a permis de mettre en évidence quatre types de trajectoires (figure 8.a): un succès de la colonisation avec une augmentation constante du nombre de groupes (courbe rouge), échec de la colonisation (courbe verte) et deux situations intermédiaires (courbes bleue et orange). La figure de droite montre la relation entre trois indicateurs de réussite de la colonisation, l'étendue de l'espace colonisé (en abscisse), la capacité à traverser la mer (en ordonnée) et le nombre de groupes (taille des cercles) à la fin de la simulation. On observe l'émergence de trois types principaux de colonisation: - les points rouges et orange illustrent la combinaison entre une démographie importante, une colonisation étendue à tout le continent $^{76}$ et une faible proportion de groupes sur l'île (Type I) ; - les points bleus et verts correspondent à une faible démographie (petite taille des cercles) et à une colonisation peu étendue spatialement, que ce soit sur l'île (c'est le cas des points en haut, Type II) ou sur un petit périmètre du continent, d'où la très grande dispersion de l'indicateur «distance moyenne » (Type III). Ces trois types peuvent être interprétés comme les trois principaux «attracteurs » des dynamiques du système de peuplement. Certaines trajectoires débouchent sur des situations intermédiaires (environ 20\% sont en dehors des cercles tiretés de la figure 8.b), ce qui illustre une certaine hétérogénéité de comportements autour des principaux attracteurs.

(Fig. 8)

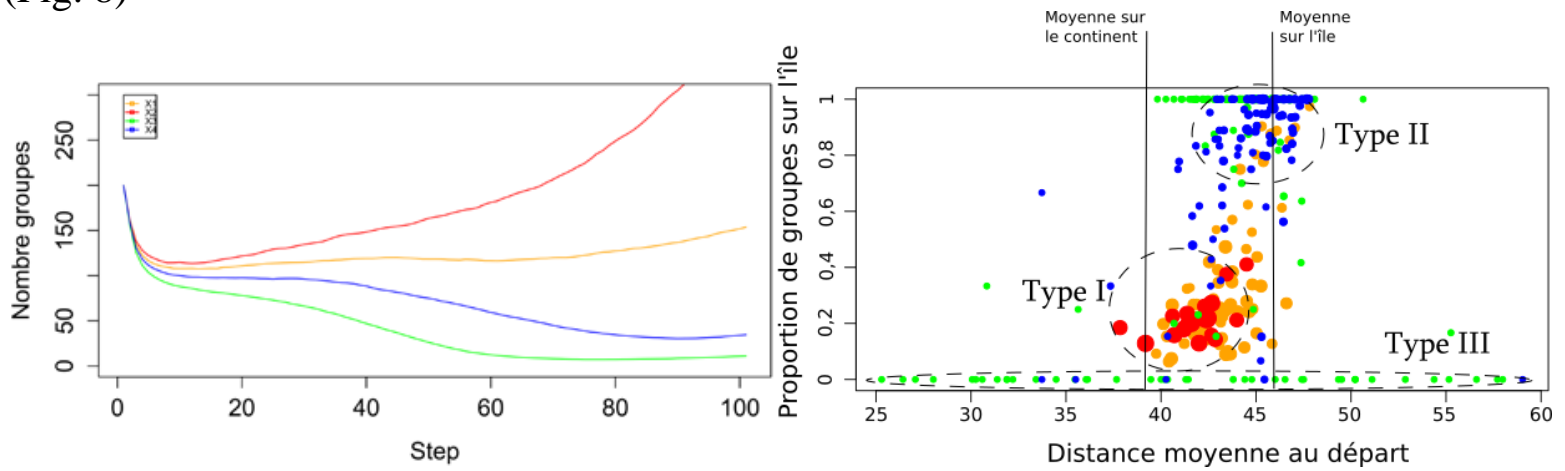

Figure 8 : Capacité du modèle à produire une diversité de trajectoires (300 réplications d'un même jeu de paramètres choisi comme référence).

\section{Discussion et perspectives}

L'approche par simulation représente un véritable intérêt pour explorer les dynamiques possibles du système de peuplement dans le passé, dans un contexte où les observations sont peu fréquentes et très hétérogènes dans le temps et dans l'espace. Il n'est alors pas possible de calibrer de façon précise une simulation puisque les données sont manquantes ou imprécises,

\footnotetext{
${ }^{76} \mathrm{Vu}$ la forme du continent virtuel et la localisation de l'île (encadré 1), la valeur moyenne de l'indicateur " distance moyenne au départ ", sous l'hypothèse d'une occupation uniforme de l'espace par les agentsgroupes, est de 39 pour le continent et de 46 pour l'île.
} 
et la validation a ici été faite «à dires d'expert ». En revanche le modèle constitue un outil d'aide à la réflexion et il permet d'explorer les effets de différentes hypothèses relatives aux comportements de groupes de chasseurs-collecteurs face à l'environnement et à leurs interactions et de dire "comment» s'est faite la colonisation d'un nouveau territoire. L'approche se veut stylisée et le modèle ne vise pas à reproduire au plus près la réalité historique. En revanche, son intérêt est de suggérer des effets non-linéaires, pas toujours intuitifs. Les résultats du modèle HU.M.E. ont permis de réfléchir à des questions concrètes, par exemple : combien de fois une colonisation a-t-elle été tentée avant de réussir ? Quelle est la part de la stochasticité et de processus connus pour expliquer succès et échec ?

Certains mécanismes introduits dans le modèle sont classiques. Tel est le cas de la rareté des ressources comme élément déclencheur d'une migration. D'autres sont plus spécifiques, comme le mécanisme de diffusion de l’innovation par proximité géographique. Ce modèle, classique en géographie, n’a pas de support empirique dans les périodes traitées ici. De même, la mobilisation de l'énergie ne correspond pas à un concept utilisé classiquement pour cette période. En revanche les travaux de Nonaka et Holme ${ }^{77}$, soulignant l'intérêt d'un SMA pour étudier les déplacements d'agents recherchant des ressources dans un paysage hétérogène, accordent une place centrale à l'énergie dans leur modèle. Celui-ci ne concerne pas des groupes humains mais, sur un plan conceptuel, la situation est la même que dans le modèle HU.M.E. (des ressources hétérogènes qui se régénèrent et des prédateurs qui se déplacent et qui consomment). Dans ce contexte des chasseurs-cueilleurs il y a 70000 ans, il n'y a pas de stockage et le concept d'énergie est plutôt utilisé de manière métaphorique pour traduire l'hypothèse qu'un groupe restant un certain temps dans une même zone géographique a pu bénéficier d'un temps de préparation pour organiser une traversée par exemple.

A côté du déplacement lié à la rareté des ressources, il existe dans le modèle une probabilité faible de "déplacement sans raison ». Cette règle a été suggérée par les thématiciens du groupe afin d'appréhender d'autres logiques de déplacement, très probablement présentes mais difficiles à préciser. Ces différentes logiques n'ont pas été oubliées et ont été regroupées par souci de simplification sous une même catégorie dont le label "partir sans raison » signifie simplement "sans raison de rareté de la ressource ». Une multitude de bonnes « raisons » peuvent en effet être imaginées dans le monde « réel ».

Le modèle HU.M.E. est appelé à d'autres développements. Dans sa version actuelle, il sera utilisé pour explorer plus en détail le rôle que joue le mode de diffusion de l'innovation technique dans la réussite d'une colonisation. Par ailleurs, le modèle pourra être enrichi en dotant les agents de traits génétiques, ce qui permettra de suivre leur généalogie (entre autres pour inscrire les distributions de gènes dans une structure de transmission) et de confronter les résultats des simulations à ceux fournis par les généticiens (différence arbres phylogénétiques / vraies filiations).

\footnotetext{
77 Nonaka Estuko, HolmePetter, « Agent-based model approach to optimal foraging in heterogeneous landscapes: Effects of patch clumpiness », Ecography, 30(6), 2007, p. 777-788.
} 